Discussant: P. Bomba, Excellus BlueCross BlueShield \& MedAmerica Insurance Company, Rochester, New York

The topic of end-of-life (EOL) care planning has received a surge of attention in recent years. Programs designed to ensure that care preferences are appropriately implemented in actual care when a person is facing a serious advancing illness have been a high priority, with a particular focus on improving how preferences are communicated across care settings. However, both implementation and educational challenges have emerged. The purpose of this symposium is to explore the process of determining patient EOL care preferences in a variety of care settings, as well as to evaluate the role of educational resources that can be drawn upon to support this process. The first three studies all focus on issues around the implementation of the MOLST (Medical Orders for Life-Sustaining Treatment) in Massachusetts. Chen and colleagues focus on MOLST completion in the hospital setting, Boerner and colleagues examine how nursing home staff experience the MOLST process, and DeSanto-Madeya and colleagues highlight the voice of key stakeholders in identifying implementation challenges and training and education needs. The final presentation by Gazarian focuses more generally on the capacity of educational resources and decision aids in supporting both patients and health care providers as they engage in thoughtful EOL discussions. The discussion will reflect on the shared experiences and develop a dialogue about ways to overcome the identified implementation and educational challenges.

\section{THE RELATIONSHIP BETWEEN DISEASE BURDEN, CARE SETTING, AND LIFE-SUSTAINING TREATMENT CHOICES}

E. Chen ${ }^{1}$, C.T. Pu, MD², J. Ragland, $\mathrm{MD}^{3}$, J. Schwartz, $\mathrm{MD}^{4}$, M. Fairbanks, BS ${ }^{5}$, J.E. Mutchler ${ }^{6}, 1$. Massachusetts Department of Public Health, Boston, Massachusetts, United States, 2. Partners HealthCare, Boston, MA, USA, 3. Partners HealthCare, Boston, MA, USA, 4. Partners HealthCare, Boston, MA, USA, 5. Partners HealthCare, Boston, MA, USA, 6. University of Massachusetts Boston, Boston, MA, USA

Life-sustaining treatment preferences were collected from 593 Medical Orders for Life-Sustaining Treatment forms (MOLST) at three hospitals. Logistic regression models estimated the odds for choosing All Treatment (aggressive) vs. Limitations to Treatment for patients with high illness burden (Charlson Score $>5$ vs. $\leq 5)$, controlling for decision-maker (patient/proxy), clinician type, and patient demographics. Over one-third (36\%) chose All Treatment. Mean Charlson Score was $8(\mathrm{SD}=3)$. Proxy decision-makers signed $43 \%$ of the forms. Mean patient age was $71(\mathrm{SD}=15) ; 49 \%$ were male, and $83 \%$ were non-Hispanic White. Palliative care clinicians administered $50 \%$, and both non-palliative and palliative MDs administered $52 \%$ of the MOLSTs. Patients with Charlson $>5$ were $70 \%(\mathrm{OR}=0.31 ; \mathrm{p} \leq 0.001)$ less likely to choose All Treatment than those with Charlson $\leq 5$, controlling for only patient characteristics (pseudo R2 $=0.148$; $\mathrm{p} \leq 0.001)$. Care settings with palliative care clinicians attenuated the magnitude of the relationship between illness burden and life-sustaining treatment preferences $(\mathrm{OR}=0.54$; $\mathrm{p} \leq 0.10$ )(pseudo $\mathrm{R} 2=0.431 ; \mathrm{p} \leq 0.001$ ).
IMPLEMENTING THE MOLST (MEDICAL

ORDERS FOR LIFE-SUSTAINING TREATMENTS): CHALLENGES FACED BY NURSING HOME STAFF

K. Boerner ${ }^{1}$, J. Rodriquez, $\mathrm{PhD}^{2}$, E. Quach, $\mathrm{PhD}^{3}$, M. Hendricksen, $\mathrm{MPH}^{4}, 1$. University of Massachusetts Boston, Boston, Massachusetts, United States, 2. University of Massachusetts Boston, Department of Sociology, Boston, MA, USA, 3. Center for Healthcare Organization and Implementation Research, Bedford VA Medical Center, Bedford, MA, 4. University of Massachusetts Boston, Department of Gerontology, Boston

This study examined how the Medical Order for Life-sustaining Treatment (MOLST) is implemented in two nursing homes in Massachusetts; one had primarily long-term care residents and high hospice utilization, the other had low hospice utilization and a high proportion of post-acute care residents. Qualitative in-person interviews with 21 staff members who had a role implementing the MOLST explored their experiences using the form in their daily work routines. Staff at both nursing homes described benefits of the MOLST such as providing guidance for staff and family. Yet, they also gave detailed accounts of challenges they face in implementing the form. They reported problems with the form itself such as confusing language and conflicting categories as well as a set of procedural challenges that undermined the timely completion of the form. The nursing home with more post-acute care residents faced more challenges with transferability of the MOLST to and from hospitals.

\section{ENGAGING KEY STAKEHOLDERS TO IMPROVE THE MOLST (MEDICAL ORDERS FOR LIFE-SUSTAINING TREATMENTS) PROCESS}

S. DeSanto-Madeya ${ }^{1}$, C. Abrahamson, $\mathrm{BSN}^{2}$, K. DiGuglelmo, MSN (c) $)^{3}$ 1. Boston College, Chestnut Hill, Massachusetts, United States, 2. Boston College, Connell School of Nursing, Chestnut Hill, MA, USA, 3. Boston College, Connell School of Nursing, Chestnut Hill, MA, USA

Six MOLST - Meeting the Challenge workshops were held with key interdisciplinary stakeholders from across the state of Massachusetts to start a dialogue on the challenges encountered in the implementation of MOLST. Three hundred and fifty-eight key stakeholder, including nurses, nurse practitioners, physicians, physician assistants, social workers, clergy, lawyers, and EMS providers completed a survey at the completion of the workshop. The majority of respondents believe the following is needed: interdisciplinary training and education for all providers on healthcare planning using common language and tools $(90 \%)$, the MOLST process needs to be clarified and the form easily accessible (89\%), the MOLST form be redesigned $(81 \%)$, an e-MOLST state registry be implemented $(76 \%)$, and a MOLST outcome monitoring system be established $(71 \%)$. These findings will help to craft a responsive MOLST process that honors the goals and preferences of persons living with serious illness in all Massachusetts health care settings. 